\title{
23. A Note on the Affine Difterential Geometry of a Surface.
}

By Buchin Su.

(Received July 13, 1932.)

1. Let us consider a surface $S$, a curve $C$ on $S$, and three neighbouring points $P, P_{1}, P_{2}$, on $C$. The three tangents at these points to the asymptotic curves of one system determine a quadric whose limit, as $P_{1}, P_{2}$ independently approach $P$ along $C$, is a quadric called by Bompiani $\left({ }^{1}\right)$ an asymptotic osculating quadric of $C$ at $P$. A second asymptotic osculating quadric is obtained by using the other system of asymptotics. We shall now derive the equations of these quadrics, using Blaschke's notations, and shall deduce some of their affine-geometrical properties in connection with the geometrical significances of the affine normal, the tangents of Darboux, the tangents of Segre, the elementary forms of Bompiani and the projective line-element of Fubini at a point of a surface.

2. Let the coordinates $x$ of a point $P$ on a non-degenerate nondevelopable surface $S$ be given as analytic functions of two independent variables $u, v$; and let the curves $u=$ const. $v=$ const. be the asymptotics. Then the functions $x$ are solutions of a system of differential equations,

(1)

$$
\mathfrak{x}_{u u}=\frac{F_{u}}{F} \mathfrak{x}_{u}+\frac{A}{F} \mathfrak{x}_{v}
$$

$$
\begin{aligned}
& \mathfrak{x}_{u v}=F_{\mathfrak{y}}, \\
& \mathfrak{x}_{v v}=\frac{D}{F} \mathfrak{x}_{u}+\frac{F v}{F} \mathfrak{x}_{v} ;
\end{aligned}
$$

$$
\mathfrak{n}_{u}=-H_{\mathfrak{x}_{u}}+\frac{A_{\mathfrak{i}^{\prime}}}{F^{2} \mathfrak{w}_{v}}
$$

(1) E. Bompiani, La geometria delle superficie considerate nello spazio rigato, Rendiconti dei Lincei, (VI) 3 (1926), pp. 395-400; Ancora sulla geometia...., Rendiconti dei Lincei, (VI) 4 (1926), pp. 262-267; Eín Analogon der Quadrik von Lie in der projektiven Flächentheorie, Math. Zeits., 29 (1929), pp. 678-683. Also see E. P. Lane, The asymptotic osculating quadrics of a curve on a surface, Bull. Amer. Math. Soc., 33 (1927), pp. 195-200. 


$$
\mathfrak{y}_{v}=+\frac{D_{u}}{F^{2}} \mathfrak{x}_{u}-H_{\mathfrak{x}_{v}}
$$

The one-parameter family of curves on $S$ represented by the equation

$$
d v-\lambda d u=0
$$

contains one curve $C$ through $P$. The coordinates $z$ of a point of the ruled surface $R^{(u)}$ formed by the tangents drawn to the asymptotics of system $u(d v=0)$ from every point of $C$ are of the form

$$
z(u, w)=\mathfrak{x}+w \mathfrak{x}_{u},
$$

where the variable $v$ in $\mathfrak{x}, \mathfrak{x}_{u}$ is to be considered, after (3), as a function of $u$.

Differentiating (4) and reducing by means of (1), (2) and (3), we get

$$
\begin{aligned}
\mathfrak{z}_{u}= & \left(1+w \frac{F_{u}}{F}\right) \mathfrak{x}_{u}+\left(\lambda+w \frac{A}{F}\right) \mathfrak{x}_{v}+\lambda w F \mathfrak{y}, \\
\mathfrak{z}_{w}= & \mathfrak{x}_{u,} \\
\mathfrak{z}_{u u}= & \left({ }^{*}\right) \mathfrak{x}_{u}+\left[\frac{A}{F}+\lambda^{\prime}+\lambda^{2} \frac{F_{v}}{F}+w\left(\frac{A u}{F}+2 \lambda \frac{A u}{F^{\prime}}-\lambda^{2} H F\right)\right] \mathfrak{x}_{u}, \\
& +\left[2 \lambda F^{\prime}+w\left(A+2 \lambda F_{u}+\lambda^{2} F_{v}+\lambda^{\prime} F^{\prime}\right)\right] \mathfrak{y}, \\
\mathfrak{z}_{u w}= & \frac{F_{u}}{F^{\prime}} \mathfrak{x}_{u}+\frac{A}{F} \mathfrak{x}_{v}+\lambda F \mathfrak{y},
\end{aligned}
$$

where $\lambda^{\prime}$ is the total derivative of $\lambda$. The differential equation of the curved asymptotics of $R^{(u)}$,

$$
\left(z_{u u}, z_{u}, z_{w}\right) d u+2\left(\jmath_{w} u, z_{u}, z_{w}\right) d w=0,
$$

is now reduced to the form

(5) $1+\frac{d w}{d u}=-\frac{1}{\lambda} w\left(\frac{A}{F}+\lambda \frac{F u}{F^{2}}\right)+\frac{1}{2} \frac{w^{2}}{\lambda^{2}}\left[-\left(\frac{A}{F}\right)^{2}+\lambda \frac{A}{F} \frac{\partial}{\partial u} \log \left(A \mid F^{2}\right)\right.$

$$
\left.+\lambda^{2} \frac{A}{F} \frac{\partial}{\partial v} \log \left(A^{2} \mid F\right)-\lambda^{3} H F-\lambda^{\prime} \frac{A}{F}\right] \text {. }
$$

The coordinates of a point on the tangent drawn from the point $z$ to the curved asymptotic of $R^{(u)}$, which passes through $z$, are evidently of the form

$$
\bar{z}(w, \mu)=z+\mu \frac{d z}{d u},
$$

where $\mu$ is another parameter and the variable $w$ is to be considered, 
after (5), as a function of $u$. If we express, as it is always possible, the coordinates of any point in space in the form

$$
\mathfrak{x}+x \mathfrak{x}_{u}+y \mathfrak{x}_{v}+z \mathfrak{n}
$$

then the local coordinates $x, y, z$ of the point (6) are given by the equations,

$$
\begin{aligned}
& x=w+\mu\left(1+\frac{d w}{d u}+w \frac{F_{u}}{F}\right), \\
& y=\mu\left(\lambda+w \frac{A}{F}\right), \\
& z=\lambda F u \mu .
\end{aligned}
$$

Substituting (5) in the first equation of (7) and eliminating $\mu, w$ from (7), we get the equation to the osculating quadric of $R^{(u)}$ along the asymptotic tangent $u$ at $P$,

$$
\begin{aligned}
& \lambda^{3}\left(H z^{2}-2 z+2 F x y\right)-2 \frac{A}{F} \lambda^{2} x^{\prime} z+2 \frac{A}{F^{7}} \lambda y z \\
& -\frac{A}{F^{2}}\left\{\frac{A}{F}+\lambda \frac{A u}{A}+\lambda^{2} \frac{\partial}{\partial v} \log \left(A^{2} / F^{\prime}\right)-\lambda^{\prime}\right\} z^{2}=0,
\end{aligned}
$$

which is nothing but the equation of the first asymptotic osculating quadric $Q^{(u)}$ of $C$ at $P$. The equation of the second asymptotic osculating quadric $Q^{(r)}$ of $C$ at $P$ is

(9)

$$
\begin{aligned}
H z^{2} & -2 z+2 F x y+2 \frac{D}{F} \lambda^{2} x z-2 \frac{D}{F} \lambda y z \\
& -\frac{D}{F^{2}} \lambda\left\{\frac{D}{F^{3}} \lambda^{2}+\frac{D_{n}}{D} \lambda+\frac{\partial}{\partial u} \log \left(D^{2} \mid F\right)+\lambda^{\prime}\right\} z^{2}=0 .
\end{aligned}
$$

3. The diameter of $Q^{(u)}$ which passes through $P$ is given by the equations

$$
x: y: z=-\frac{A}{F^{2} \lambda^{2}}: \frac{A}{F^{2} \lambda}: 1
$$

Similarly, the diameter of $Q^{(v)}$ which passes through $P$ is of the equations

$$
x: y: z=\frac{D}{F^{2}} \lambda:-\frac{D}{F^{2}} \lambda^{2}: 1 \text {. }
$$

Since these diameters are independent of $\lambda^{\prime}$, we may conclude that the centres of the asymptotic osculating quadrics of one family of curves which are tangent to each other at a surface point $P$ lie on a line passing through $P$. 
Such a line we will call the line of centres of this family corresponding to the common direction of the curves at $P$.

Eliminating $\lambda$ from the equations (10) we get

$$
A x z+F^{2} y^{2}=0
$$

which represents a quadratic cone $C^{(u)}$. Similarly, from (11) we also obtain another quadratic cone $C^{(v)}$ of the equation

$$
D y z+F^{2} x^{2}=0 \text {. }
$$

Thus we get:

When the tangent varies in the pencil with centre $P$, the corresponding line of centres of one family describes a quadratic cone touching the tangent plane of the surface at $P$ along one of the asymptotic tangents.

These cones have once appeared in the investigations of the quadrics of Moutard, and are determined by the three cuspidal lines of a certain cone $\Gamma_{4}$, i. e., the locus of the diameter of the quadric of Moutard passing through $P$, the affine surface-normal and one of the asymptotic tangents $\left({ }^{2}\right)$.

The plane containing the lines (10) and (11) is given by the equation

$$
\left|\begin{array}{ccc}
x, & y, & z \\
-\frac{A}{F^{2} \lambda^{2}}, \frac{A}{F^{22} \lambda}, & 1 \\
\frac{D}{F^{2}} \lambda, & -\frac{D}{F^{2}} \lambda^{2}, & 1
\end{array}\right|,
$$

or

$$
\left(A+D \lambda^{3}\right)(y+\lambda x)=0 .
$$

This becomes indeterminate when, and only when the tangent is a tangent of Darboux. Thus we get a new characterization of the direction of Darboux: The directions of Darboux are the directions of curves whose asymptotic osculating quadrics of both families have a common line of centres $\left({ }^{3}\right)$.

(2) Cf. my papers: The quadrics of Moutard, I, II, Tohoku Math. Journ., 33 (1930), pp. 26-38; pp. 190-198. W. Haack has also gotten these cones from the standpoint of the line geometry; cf. his paper: Affine Differentialgeometrie der parabolischen Strahlensysteme, Math. Zeits., 33 (1931), §8, p. 260.

( $\left.{ }^{3}\right)$ This can be derived from a theorem of Bompiani (cf. Fubini-Čech, Geometria proiettiva differenziale, vol. II, Appendix 2) and conversely; or Haack, loc. cit., Satz IX. 
In other cases these lines of centres determine a plane $\pi_{\lambda}$ corresponding to a tangent $t_{\lambda}$ :

$$
y+\lambda x=0
$$

which passes through the conjugate tangent of $t_{\lambda}$ and the affine normal.

Thus we get a geometrical significance of the affine normal:

The affine surface normal at a point $P$ is the caxis of a pencil of planes, each of them being determined by the two lines of centres corresponding to every tangent of the sunface at $P$.

4. The quadric $Q^{(u)}$ becomes the quadric of Lie, whose equation is

$$
H z^{2}-2 z+2 F x y=0
$$

in case $\lambda \rightarrow \infty$, while $Q^{(v)}$ becomes the quadric of Lie in case $\lambda=0$.

The conjugate line of (10) with respect to the quadric of $\mathrm{Lie}$ envelopes a parabola $C_{1}$ in the tangent plane $z=0$. This parabola is tangent to the asymptotic tangent $z=x=0$ at $P$ and has the other asymptotic tangent for its diameter. Its equation is easily found, i.e.,

$$
z=A x^{2}-4 F y=0 \text {. }
$$

Similarly, we have another parabola which is the envelope of the conjugate line of (11) with respect to the quadric of Lie and whose equation is

$$
z=D y^{2}-4 F x=0 \text {. }
$$

These parabolas intersect, besides at $P$, also at three points, $P_{1}, P_{2}$, $P_{3}$ which lie on the tangents of Segre,

$$
A x^{3}-D y^{3}=0 \text {. }
$$

The coordinates of $P$, are

$$
x=\frac{4 \varepsilon^{r} F}{A \alpha^{2}}, y=\frac{4 \varepsilon^{2 r} F}{A \alpha}, z=0,
$$

where $\alpha=\sqrt[3]{A \mid D}, \varepsilon=\sqrt[3]{1} ; r=1,2,3$. These points are vertices of a triangle whose centroid is $P$ and whose sides are parallel to the tangents of Darboux $\left({ }^{4}\right)$.

5. Let us confine ourselves to the two parabolas:

$$
x^{2}-k \frac{F}{A} y=0, y^{2}-k \frac{F}{D} x=0 \text {. }
$$

( $\left.{ }^{4}\right)$ A similar but more complicated result has been established in my paper: On the cubic indicatrices of a surface, Science Reports of Tohoku Imp. Univ., (I) 19 (1930), pp. $699-702$. 
When $k=4$, they are the parabolas we have so far considered. When $k=2$, the parabolas are considered by $\breve{C e c h}\left({ }^{5}\right)$ and are defined as having a contact of the second order with one asymptotic curve passing through $P$ and having the other asymptotic tangent for its diameter. When $k=-\frac{4}{3}$, the correspoding parabolas may be seen as the osculating conics of two affine covariant cubies, passing through $P\left({ }^{6}\right)$. By means of the value of a cross ratio or of Mehmke-Segre's invariant the parabolas (20) may geometrically be defined if we make use of the three special pairs of parabolas above quoted or any one of them.

With regard to the parabolas (20) all the results established in $\$ 4$ hold without any modification.

Now consider on the surface $S$ a point $P^{\prime}$ infinitely near to $P$. The line $P P^{\prime}$ is then in the tangent plane at $P$ and intersects the parabolas (20), besides at $P$, also at two points $G_{1}, G_{2}$ respectively. It can easily be shown that

$$
\frac{A d u^{2}}{F d v}=k \frac{P P^{\prime}}{P G_{1}}, \quad \frac{D d v^{2}}{F d u}=k \frac{P P^{\prime}}{P G_{2}^{y}}
$$

provided that the infinitesimals higher than the first order be neglected. The equations (21) express the geometrical significances of the elementary forms of Bompiani( $\left.{ }^{7}\right)$.

Let $P^{*}$ be the harmonic conjugate point of $P$ with respect to $G_{1}$, $G_{2}$. From (21) we get that

$$
k \frac{P P^{\prime}}{P P^{*}}=\frac{A d u^{3}+D d v^{3}}{2 F d u d v}
$$

which is a geometrical interpretation of the projective line-element of Fibini $\left({ }^{8}\right)$.

May, 1932.

(5) Čech, Lintorno di un punto di una superficie considerato dal punto di vista proiettivo, Annali di Mat., (3) 31 (1922), p. 205 ; also see my paper: The quadries of Moutard I, loc. cit., p. 36 .

$\left({ }^{6}\right)$ Cf. My paper: The quadrics of Moutard I, loc. cit., p. 35.

( $\left.{ }^{7}\right)$ Bompiani, Le forme elementari e la teoria proiettiva delle superficie, Bollettino delle Unione Matematica Italiana, 5 (1926), pp. 167-173 and pp. 209-214.

$\left({ }^{8}\right)$ Other significances of the projective line-element are found from the same point of view in my paper: On the cubic indicatrices of a surface, loc. cit. 